\title{
A PRACTICAL APPROACH TO PERFORM THE ISOTROPY TEST FOR EXTREMITY DOSEMETERS
}

\begin{abstract}
The requirements for determining extremity dosemeter performance have evolved over the last decade. In 2010, the Spanish Nuclear Safety Council (CSN) adopted a national protocol with performance requirements based on ISO 12794 . Because of the lack of symmetry of ISO 4037-3 calibration phantoms, the isotropy test set-up in the sagittal plane presented several challenges both for individual monitoring services and for calibration labs. This paper proposes and validates a practical approach to reduce the number of irradiations. Results show that our wrist and ring dosemeters meet the ISO 12794 requirements for the isotropy test. However, additional studies would be needed to verify the newer IEC 62387 Standard.
\end{abstract}

\section{INTRODUCTION}

The requirements for determining extremity dosemeter performance have evolved over the last decade. The ISO 4037-3 Standard ${ }^{(1)}$ introduced specific phantoms for ring and wrist dosemeter calibration. In 2000, ISO $12794^{(2)}$ provided performance criteria and tests for extremity dosemeters. These requirements were adopted by the Spanish Nuclear Safety Council (CSN) in a national protocol in $2010^{(3)}$. In 2012 our individual monitoring service (IMS) undertook the characterization of extremity dosemeters following ISO 12794 and its Spanish equivalent. Our IMS has been accredited by the Spanish Accreditation Body (ENAC) according to the ISO $17025^{(4)}$ Standard and it has also been authorized by the Spanish regulator (CSN) to perform official personal dose records. Later, IEC published a new standard $^{(5)}$ which includes new requirements for several performance tests.

The aims of this paper are:

- To verify the isotropy test of our extremity dosemeters (wrist and ring) according to ISO 12794.

- To propose a practical approach to reduce the number of irradiations.

- To use one of the set-ups for a preliminary check of IEC 62387 requirements for the isotropy test.

The performance of the isotropy test for extremity dosemeters presents different challenges not only for the IMS, but also for the secondary standard calibration laboratory (SSDL). For some of the required conditions, such as sagittal plane tests, there are no conversion coefficients published in the standard to calculate the reference quantity and some specific set-ups had to be developed.

\section{MATERIALS AND METHODS}

\section{ISO 12794 isotropy test and requirements}

According to ISO 12794, the isotropy test of ring or wrist dosemeters must be performed in units of personal dose equivalent, $H_{\mathrm{p}}(0.07)$, using a rod or a pillar phantom with photons of energy ( $\left.60 \pm 5 \mathrm{keV}\right)$. Four groups of dosemeters are required for normal incidence, 20, 40 and 60 degrees off normal, respectively. For each group, the angle of incidence for $n$ irradiations is varied in both a positive and negative direction on two planes perpendicular to each other and to the plane of the dosemeter (angles $\alpha$ and $\varphi$ in Figure 1). $n$ is the number of dosemeters used for given setup conditions. In our study it is usually set to 5, although it can vary from 2 to 15 (the exact number is specified in Tables 2 to 4).

The evaluated values must fulfill the following requirement:

$$
0.85 \leq \frac{\sum_{i} \overline{E_{i}}}{4 \overline{E_{1}}} \pm I \leq 1.15
$$

where

$\overline{E_{i}}$ is the mean of the $n$ evaluated values for group $i$,

$\overline{E_{1}}$ is the mean value of the $n$ ' dosemeters for normal incidence. 
I is the $95 \%$ confidence interval of the function $\frac{\sum_{i} \overline{E_{i}}}{4 \overline{E_{1}}}$

\section{Test set-up}

Figure 2 shows an example of the tested ring and wrist dosemeters. The ring dosemeter contains 2 detectors, one on top of the other, so that they have a filtration of 30 and $265 \mathrm{mg} \cdot \mathrm{cm}^{-2}$, respectively. The diameter of the holder that contains the dosemeters is $7.7 \mathrm{~mm}$. The wrist dosemeter consists of a plastic badge $\left(39.7 \times 36.1 \times 7.7 \mathrm{~mm}^{3}\right)$ with two detectors and a filter of 273.2 and $386.7 \mathrm{mg} \cdot \mathrm{cm}^{-2}$, respectively.. The detectors used were Bicron TLD-100 chips of 3 x 3 and $0.9 \mathrm{~mm}^{3}$. Reading was done in a Rados Reader for $20 \mathrm{~s}$ with an exponential temperature ramp up to $350{ }^{\circ} \mathrm{C}$ without annealing.

Irradiations were performed at the UPC SSDL. The irradiation distance was $2.0 \mathrm{~m}$. Following ISO 12794 requirements, the reference calibration quality was $\mathrm{N}-80^{(6)}$ (mean energy $60 \mathrm{keV}$ ) and the ISO 4037-3 rod and pillar irradiation phantoms were employed. The isotropy test for the axial plane has no special difficulty and the standard set-up is used. In the case of the sagittal plane, a special set-up was designed. Figure 3 illustrates the irradiation for $\varphi$ $=40$ for rings.

$H_{\mathrm{p}}(0.07)$ was calculated as the reference air kerma multiplied by the $H_{\mathrm{p}}(0.07)$ air kerma conversion coefficients for the axial plane ${ }^{(2)}$. For the sagittal plane the evaluation was performed in air kerma units since the conversion coefficients were not available in the Standard. Because of the symmetry of the dosemeters, only positive directions were used in the tests. Irradiations were performed independently with different dosemeters for the axial and sagittal planes.

One of the purposes of the study is to reduce the number of required irradiations by the simultaneous irradiation of several dosemeters. Before doing so, two effects were analyzed: the perturbation in the dispersion factors due to adjacent dosemeters and the difference in the focus distance for the dosemeters out of the reference point on sagittal irradiations. The dimensions of the dosemeters influence in both aspects. To study the influence of simultaneous irradiation, the mean values of 3 wrist and 5 ring dosemeters irradiated at a time were compared with the mean value of 3 wrist and 5 ring dosemeters irradiated one by one. In the case of the sagittal plane tests (angle $\varphi$ ), a correction factor, $F_{G}$, that corrects for the inverse square distance law, is introduced to take into account the distance between the different dosemeters and the focus.

$$
F_{G}=\frac{K_{a i r, d r e f}}{K_{a i r, d}}=\frac{d^{2}}{d_{r e f}^{2}}
$$

The effect of performing simultaneous irradiations, in the sagittal plane, was analyzed by calculating the ratio between the reading of the dosimeter at the reference point, $\mathrm{M}_{\text {ref }}$ (center of phantom) and the reading of the dosimeter off the reference point $\mathrm{M}_{\mathrm{d}}$ corrected by $\mathrm{F}_{\mathrm{G}}$.

After these preliminary verifications, isotropy test measurements were performed for the two angular directions $\alpha$ and $\varphi$, for $0^{\circ}, 20^{\circ}, 40^{\circ}$ and $60^{\circ}$, independently.

\section{RESULTS}

\section{Preliminary tests to validate simultaneous irradiation}

The perturbation of adjacent dosemeters was proven to be less than $1 \%$ when the distance between rings was greater than $1 \mathrm{~cm}$ between centers $(0.5 \mathrm{~cm}$ between walls) and equal or greater than $4 \mathrm{~cm}$ between centers $(1 \mathrm{~cm}$ between walls) for wrists. These conditions were followed when performing the isotropy tests.

The influence of simultaneous irradiation of 3 wrist dosemeters for the sagittal plane rotation is presented in Table 1. 
It shows the ratio between the reading of the dosemeter at the reference point (d1-center of phantom) and the reading of the dosemeter in positions away from the reference point (top (d2) and bottom (d3) in Figure 4), corrected by $F_{G}$, for $\varphi=20,40$ and $60^{\circ}$. It is verified that, once corrected, the influence of the simultaneous irradiation in the sagittal plane is lower than $1 \%$. The mean value and standard deviation of the ratio was found to be $1.00 \pm 0.01$. However, Table 1 shows that the geometric correction for $60^{\circ}$ reaches $4 \%$ and thus cannot be neglected.

The influence of simultaneous irradiation of 5 ring dosemeters for the sagittal plane was lower than $1 \%$. In this case, the geometry correction is below $1 \%$ and is thus omitted.

\section{Isotropy test}

Tables 2 and 3 summarize the angle test performance. Each table provides details about the number of dosemeters, mean, $\bar{E}_{i}$, and standard deviation, $\mathrm{s}_{\mathrm{i}}$, of the evaluated dose and the confidence level for a given set-up, I. Measurements are normalized to the $H_{\mathrm{p}}(0,07)$ used for each angular direction. Equation 1 is calculated considering only the axial rotation, only the sagittal rotation and the combination of the two as required by ISO 12794 . It can be seen that the requirements are verified both for rings and wrists.

In IEC 61267 standard, the angle of incidence requirements are defined in combination with the radiation energy response. Tests are to be performed on a phantom at $60^{\circ}$ for the three lowest energies in the whole rated range of energy. The relative response for the different energies shall be within $-29 \%$ to $+67 \%$ compared with incident irradiation with a reference radiation quality:

$0.71-U_{C, \mathrm{com}} \leq\left(\frac{\overline{E_{i}}}{\overline{E_{r, 0}}} \pm U_{C, \mathrm{com}}\right) \frac{C_{r, 0}}{C_{i}} \leq 1.67+U_{C, \mathrm{com}}$

Where:

$\overline{E_{i}}$ is the mean of the $n$ evaluated values for group i

$\overline{E_{r, 0}}$ is the mean of the evaluated values for a references energy

$\mathrm{C}_{\mathrm{r}, 0}$ is the conventional true value for the reference energy, and angle $0^{\circ}-\mathrm{C}_{\mathrm{i}}$ is the conventional true value for group $i$ $\mathrm{U}_{\mathrm{c} \text {, com }}$ is the combined relative expanded uncertainty $(\mathrm{k}=2)$ of $\mathrm{C}_{\mathrm{r}, 0} / \mathrm{C}_{\mathrm{i}}$

Taking advantage of the measurements performed in the framework of the ISO 12794, we checked the new requirement for $60 \mathrm{keV}$ and $60^{\circ}$ set-up for both angular directions. Results are shown in Table 4 and confirm that the IEC requirements are fulfilled in these conditions.

\section{CONCLUSIONS}

The ratio between the angular evaluated dose and the dose at normal incidence for the tested dosemeters was in the range $(0.98-1.00)$ for ring dosemeters and $(0.94$ - 0.97) for wrist dosemeters. In both cases the ISO 12794 requirements (0.85-1.15) are fully fulfilled.

Differences in response between angles $\alpha$ and $\varphi$ or when both irradiations are combined are negligible, within uncertainties, i.e. for the $40^{\circ}$ group the combined effect is 0.99 , while it is 1.01 for $\varphi$ and 0.97 for $\alpha$.

The validity of doing simultaneous irradiation is shown and is recommended in order to reduce the number of irradiations needed. However depending of the dimensions of the dosemeter a geometric correction factor has to be applied.

Although the IEC 62387 requirements for $60^{\circ}$ and $60 \mathrm{keV}$ have been verified, new tests will be needed for lower energies and higher angles. 


\section{REFERENCES}

1. International organization for standardization. $\mathrm{X}$ and gamma reference radiation for calibrating dosemeters and doserate meters and for determining their response as a function of photon energy - part 3: calibration of area and personal dosemeters and the measurement of their response as a function of energy and angle of incidence. ISO 4037. Geneva, 1996.

2. International organization for standardization. Nuclear energy - Radiation protection — Individual thermoluminescence dosimeters for limbs and eyes. ISO 12794. Geneva, 2000.

3. Consejo de Seguridad Nuclear. Subdirección de protección radiológica operacional. Protocolo para la caracterización y calibración de dosímetros de extremidades, ref DRM-CSN-EX05 V2. Madrid, 2010.

4. International organization for standardization. General requirements for the competence of testing and calibration laboratories. ISO 17025. Geneva, 2000.

5. International electrotechnical commission . Radiation protection instrumentation - Passive integrating dosimetry systems for environmental and personal monitoring - Part 1: General Characteristics and performance requirements. IEC/CEI 62387-1. Geneva, 2005

6. International Organization for Standardization. $\mathrm{X}$ and gamma reference radiation for calibrating dosemeters and dose rate meters and for determining their response as a function of photon energy-Part 1: Radiation characteristics and production method. ISO 4037-1. Geneva, 1996. 


\begin{tabular}{ccccc}
\hline Angle & Position & $\mathrm{d}(\mathrm{m})$ & $\mathrm{F}_{\mathrm{G}}$ & $\mathrm{M}_{\mathrm{ref}} /\left(\mathrm{M}_{\mathrm{d}} \cdot \mathrm{F}_{\mathrm{G}}\right)$ \\
\hline 20 & Top $(\mathrm{d} 2)$ & 1.98 & 0.98 & 1.01 \\
20 & Reference & 2.00 & 1.00 & 1.00 \\
20 & Bottom (d3) & 2.02 & 1.02 & 0.99 \\
40 & Top (d2) & 1.97 & 0.97 & 1.00 \\
40 & Reference & 2.00 & 1.00 & 1.00 \\
40 & Bottom (d3) & 2.03 & 1.03 & 0.98 \\
60 & Top (d2) & 1.96 & 0.96 & 1.01 \\
60 & Reference & 2.00 & 1.00 & 1.00 \\
60 & Bottom (d3) & 2.04 & 1.04 & 1.01 \\
\cline { 3 - 4 } & & & Mean & 1.00 \\
& & & Std & 0.01 \\
\hline
\end{tabular}

Table 1: Influence of simultaneous irradiation of 3 wrist dosemeters for the sagittal plane rotation.

\begin{tabular}{|c|c|c|c|c|c|c|c|c|c|c|c|}
\hline \multirow[b]{2}{*}{ i } & \multirow[b]{2}{*}{ angle } & \multicolumn{4}{|c|}{ Axial rotation $(\alpha)$} & \multicolumn{4}{|c|}{ Sagittal rotation $(\varphi)$} & \multicolumn{2}{|c|}{ Addition } \\
\hline & & $\mathrm{n}_{\mathrm{i}}$ & $\bar{E}_{i}$ & $\mathrm{~s}_{\mathrm{i}}$ & $\mathrm{I}_{\mathrm{Ei}}$ & $\mathrm{n}_{\mathrm{i}}$ & $\bar{E}_{i}$ & $\mathrm{~s}_{\mathrm{i}}$ & $\mathrm{I}_{\mathrm{Ei}}$ & $\bar{E}_{i}$ & $\mathrm{I}_{\mathrm{Ei}}$ \\
\hline 1 & 0 & 5 & 2.00 & 0.02 & 0.02 & 5 & 2.78 & 2.31 & 0.02 & 2.55 & 0.02 \\
\hline 2 & 20 & 5 & 2.01 & 0.03 & 0.04 & 2 & 2.71 & 2.31 & 0.01 & 2.55 & 0.04 \\
\hline 3 & 40 & 5 & 1.95 & 0.01 & 0.01 & 10 & 2.26 & 2.33 & 0.03 & 2.52 & 0.01 \\
\hline \multirow[t]{4}{*}{4} & 60 & 5 & 1.93 & 0.02 & 0.03 & 2 & 2.71 & 2.30 & 0.02 & 2.50 & 0.07 \\
\hline & & \multicolumn{4}{|c|}{$\mathrm{I}=0.01$} & \multicolumn{4}{|c|}{$\mathrm{I}=0.02$} & \multicolumn{2}{|c|}{$\mathrm{I}=0.01$} \\
\hline & & \multicolumn{4}{|c|}{$\sum \frac{\overline{E_{i}}}{4 \overline{E_{1}}}-I=0.98$} & \multicolumn{4}{|c|}{$\sum \frac{\overline{E_{i}}}{4 \overline{E_{1}}}-I=0.98$} & \multicolumn{2}{|c|}{$\sum \frac{\overline{E_{i}}}{4 \overline{E_{1}}}-I=0.98$} \\
\hline & & \multicolumn{4}{|c|}{$\sum \frac{\overline{E_{i}}}{4 \overline{E_{1}}}+I=1.00$} & \multicolumn{4}{|c|}{$\sum \frac{\overline{E_{i}}}{4 \overline{E_{1}}}+I=1.02$} & \multicolumn{2}{|c|}{$\sum \frac{\overline{E_{i}}}{4 \overline{E_{1}}}+I=1.00$} \\
\hline
\end{tabular}

Table 2: Measurements and calculations for rings on a rod phantom for each angular direction and for both contributions together to test if $\quad \sum \frac{\overline{E_{i}}}{4 \bar{E}_{1}} \pm I \quad$ is between 0.85 and 1.15 . 


\begin{tabular}{|c|c|c|c|c|c|c|c|c|c|c|c|}
\hline \multirow[b]{2}{*}{$\mathrm{i}$} & \multirow[b]{2}{*}{ angle } & \multicolumn{4}{|c|}{ Axial rotation $(\alpha)$} & \multicolumn{4}{|c|}{ Sagittal rotation $(\varphi)$} & \multicolumn{2}{|c|}{ Addition } \\
\hline & & $\mathrm{n}_{\mathrm{i}}$ & $\bar{E}_{i}$ & $\mathrm{~s}_{\mathrm{i}}$ & $\mathrm{I}_{\mathrm{Ei}}$ & $\mathrm{n}_{\mathrm{i}}$ & $\bar{E}_{i}$ & $\mathrm{~s}_{\mathrm{i}}$ & $\mathrm{I}_{\mathrm{Ei}}$ & $\bar{E}_{i}$ & $\mathrm{I}_{\mathrm{Ei}}$ \\
\hline 1 & 0 & 3 & 2.33 & 0.02 & 0.04 & 3 & 3.24 & 0.02 & 0.06 & 3.24 & 0.04 \\
\hline 2 & 20 & 3 & 2.30 & 0.01 & 0.03 & 4 & 3.22 & 0.04 & 0.06 & 3.21 & 0.04 \\
\hline 3 & 40 & 6 & 2.24 & 0.03 & 0.03 & 5 & 3.02 & 0.02 & 0.02 & 3.06 & 0.03 \\
\hline \multirow[t]{4}{*}{4} & 60 & 3 & 2.25 & 0.07 & 0.18 & 5 & 2.78 & 0.02 & 0.04 & 2.89 & 0.12 \\
\hline & & \multicolumn{4}{|c|}{$\mathrm{I}=0.02$} & \multicolumn{4}{|c|}{$\mathrm{I}=0.02$} & \multicolumn{2}{|c|}{$\mathrm{I}=0.01$} \\
\hline & & \multicolumn{4}{|c|}{$\sum \frac{\overline{E_{i}}}{4 \overline{E_{1}}}-I=0.94$} & \multicolumn{4}{|c|}{$\sum \frac{\overline{E_{i}}}{4 \overline{E_{1}}}-I=0.93$} & \multicolumn{2}{|c|}{$\sum \frac{\overline{E_{i}}}{4 \overline{E_{1}}}-I=0.94$} \\
\hline & & \multicolumn{4}{|c|}{$\sum \frac{\overline{E_{i}}}{4 \overline{E_{1}}}+I=0.99$} & \multicolumn{4}{|c|}{$\sum \frac{\overline{E_{i}}}{4 \overline{E_{1}}}+I=0.96$} & \multicolumn{2}{|c|}{$\sum \frac{\overline{E_{i}}}{4 \overline{E_{1}}}+I=0.97$} \\
\hline
\end{tabular}

Table 3: Measurements and calculations for wrists on a pillar phantom for each angular direction and for both contributions together to test if $\quad \sum \frac{\overline{E_{i}}}{4 \bar{E}_{1}} \pm I \quad$ is between 0.85 and 1.15 .

\begin{tabular}{ccccccccc}
\hline \multicolumn{9}{c}{ Rings } \\
\hline Angle & Low limit & {$[\mathrm{V}]_{\text {low }}$} & {$[\mathrm{V}]_{\text {high }}$} & High limit & Low limit & {$[\mathrm{V}]_{\text {low }}$} & {$[\mathrm{V}]_{\text {high }}$} & High limit \\
\hline$\alpha:$ & $0.64 \leq$ & 0.89 & 1.04 & $\leq 1.74$ & $0.64 \leq$ & 0.94 & 1.08 & $\leq 1.74$ \\
$\varphi:$ & $0.64 \leq$ & 0.92 & 1.07 & $\leq 1.74$ & $0.65 \leq$ & 0.70 & 0.85 & $\leq 1.74$ \\
\hline$\left[V_{\text {Low }}=\left(\frac{\bar{E}_{i}}{E_{r, 0}}-U_{C, \text { com }}\right)\right.$ & $\frac{C_{r, 0}}{C_{i}} ;\left[V_{\text {High }}=\left(\frac{\bar{E}_{i}}{E_{r, 0}}+U_{C, \text { com }}\right)\right.$ & $\frac{C_{r, 0}}{C_{i}} ; U_{C, \text { com }}=0.07$ & & &
\end{tabular}

Table 4: - Results of IEC 62387 isotropy test for N80 at $60^{\circ}$ (equation 3 verification). 


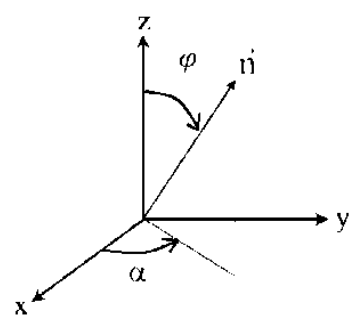

Figure 1: Planes of rotation: $\alpha$ (axial plane); $\varphi$ (sagittal plane)

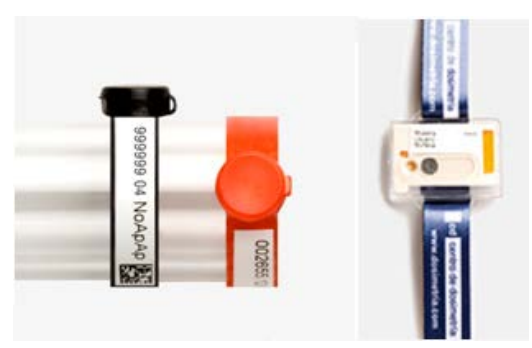

Figure 2: Rados ring and wrist dosemeters from centro de dosimetria

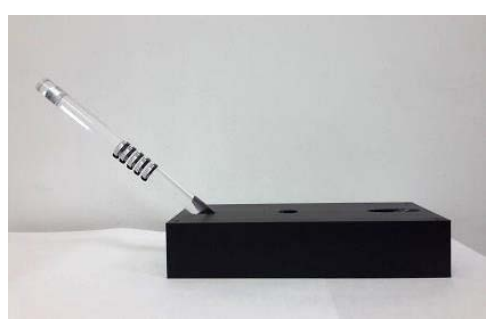

Figure 3: Isotropy test set-up for rings ( $\varphi=40)$

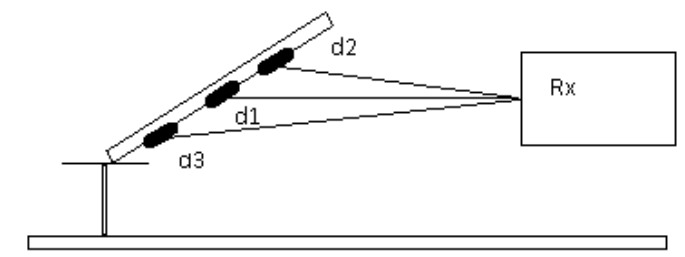

Figure 4: Arrangement for the sagittal plane rotation 